\title{
The Equation of State of Asymmetric Nuclear Matter at Zero and Finite Temperatures with the Variational Method
}

\author{
Hajime Togashi* \\ Department of Pure and Applied Physics, Waseda University, \\ 3-4-1 Okubo Shinjuku-ku, Tokyo 169-8555, Japan \\ E-mail: hajime_togashi@ruri.waseda.jp
}

\section{Hiroaki Kanzawa}

Research Institute for Science and Engineering, Waseda University, 3-4-1 Okubo Shinjuku-ku, Tokyo 169-8555, Japan

E-mail: h.kanzawa@kurenai.waseda.jp

\section{Masatoshi Takano}

Research Institute for Science and Engineering, Waseda University,

Department of Pure and Applied Physics, Waseda University,

3-4-1 Okubo Shinjuku-ku, Tokyo 169-8555, Japan

E-mail: takanomewaseda.jp

\begin{abstract}
The equation of state (EOS) of asymmetric nuclear matter at zero and finite temperatures is constructed with the variational method, starting from the realistic nuclear Hamiltonian composed of the AV18 and UIX nuclear potentials. At zero temperature, the energy per nucleon of asymmetric nuclear matter is calculated in the two-body-cluster approximation with the three-body-force contribution treated somewhat phenomenologically so as to reproduce the empirical saturation conditions. At finite temperatures, the free energies per nucleon of asymmetric nuclear matter are obtained with an extension of the variational method by Schmidt and Pandharipande. Validity of the frozen-correlation approximation employed in this study is confirmed. The obtained free energies and related thermodynamic quantities for various densities, temperatures and proton fractions are essential ingredients in our project for constructing a new nuclear EOS table for supernova simulations.
\end{abstract}

11th Symposium on Nuclei in the Cosmos

19-23 July 2010

Heidelberg, Germany.

\footnotetext{
* Speaker.
} 


\section{INTRODUCTION}

It is a challenging problem in astrophysics to elucidate the mechanism of core-collapse supernova (SN) explosion. Although many efforts have been devoted to hydrodynamic numerical simulations of $\mathrm{SN}$, the mechanism is not clarified yet. In the numerical simulations of $\mathrm{SN}$, the equation of state (EOS) of hot, dense matter is an essential ingredient. Since the EOS for SN simulations must cover an extremely wide range of densities, temperatures and proton fractions, at present, only two types of nuclear EOSs are available for SN simulations: One is constructed by Lattimer and Swesty [1], and another is by Shen et al [2]. Extensions of the Shen-EOS so as to take into account hyperon mixing [3] and hadron-quark phase transition [4] are also available.

Since these EOSs are based on phenomenological models, we undertook to construct a new nuclear EOS based on the realistic nuclear Hamiltonian with a variational method. In Refs. [5, 6], we constructed the nuclear EOS at zero and finite temperatures for uniform symmetric nuclear matter and neutron matter. In this paper, we extend the study to arbitrarily asymmetric nuclear matter.

\section{VARIATIONAL CALCULATION AT ZERO TEMPERATURE}

In this section, we construct the EOS of asymmetric nuclear matter at zero temperature. The nuclear Hamiltonian is expressed as the sum of the two-body Hamiltonian $\mathrm{H}_{2}$ and the three-body Hamiltonian $H_{3}$. The two-body Hamiltonian $H_{2}$ is defined as

$$
H_{2}=-\sum_{i=1}^{N} \frac{\hbar^{2}}{2 m} \nabla_{i}^{2}+\sum_{i<j}^{N} V_{i j}
$$

Here, $m$ is the nucleon mass and $V_{i j}$ is the two-body potential, for which the AV18 potential is chosen.

The expectation value of $\mathrm{H}_{2}$ is calculated with the Jastrow wave function

$$
\Psi=\operatorname{Sym}\left[\prod_{i<j} f_{i j}\right] \Phi_{\mathrm{F}},
$$

where $\Phi_{\mathrm{F}}$ is the Fermi-gas wave function at zero temperature. The correlation function $f_{i j}$ in Eq. (2.2) consists of spin-isospin-dependent central correlation functions $f_{\mathrm{Cts}}^{\mu}\left(r_{i j}\right)$, tensor correlation functions $f_{\mathrm{T} t}^{\mu}\left(r_{i j}\right)$ and spin-orbit correlation functions $f_{\mathrm{SO} t}^{\mu}\left(r_{i j}\right)$. Here, $s$ is the two-body total spin, $t$ is the two-body total isospin, and $\mu$ is the third component of $t$, respectively. It is noted that $\mu$ distinguishes the three isospin-triplet pairs, i.e., p-p, p-n and n-n.

In this study, the expectation value of $H_{2}$ is expressed in the two-body cluster approximation, and minimized with respect to the correlation functions to obtain the two-body energy $E_{2} / N$. In this minimization, the following two constraints are imposed: One is the extended Mayer's condition expressed as

$$
4 \pi \rho \int_{0}^{\infty}\left[F_{t s}^{\mu}(r)-F_{\mathrm{Fts}}^{\mu}(r)\right] r^{2} d r=0,
$$

where $F_{t s}^{\mu}(r)$ is the spin-isospin-dependent radial distribution function, and $F_{\mathrm{F} t s}^{\mu}(r)$ is $F_{t s}^{\mu}(r)$ in the case of the Fermi gas. This is a certain kind of the normalization condition. 


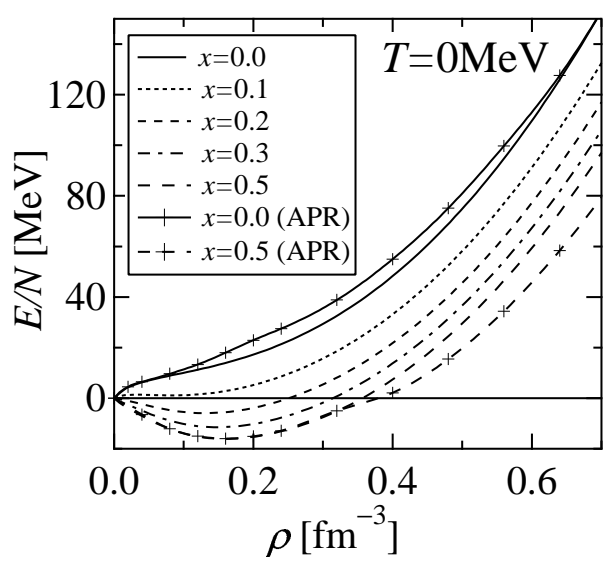

Figure 1: Energies per nucleon for various proton fractions $x$ as functions of the density $\rho$. Energies obtained with the FHNC calculation (APR) for symmetric nuclear matter and neutron matter are also shown.

The other is the healing-distance condition, which is expressed as

$$
f_{\mathrm{C} t s}^{\mu}(r)=1, \quad f_{\mathrm{T} t}^{\mu}(r)=0, \quad f_{\mathrm{SO} t}^{\mu}(r)=0 . \quad\left(r \geq r_{\mathrm{h}}\right)
$$

Namely, the correlations vanish at any distance between two nucleons $r$ larger than the healingdistance $r_{\mathrm{h}}$. The healing distance $r_{\mathrm{h}}$ is determined in Ref. [5] so that the obtained $E_{2} / N$ for symmetric nuclear matter is close to the result with a more sophisticated variational method, i.e., the Fermi Hypernetted Chain (FHNC) calculations, by Akmal et al. (APR) [7].

The contribution from the three-body Hamiltonian $\mathrm{H}_{3}$, with use of the UIX three-body nuclear potential, is expressed as

$$
\frac{E_{3}}{N}(\rho, x)=\alpha \frac{E_{3}^{\mathrm{R}}}{N}(\rho, x)+\beta \frac{E_{3}^{2 \pi}}{N}(\rho, x)+\gamma \rho^{2} e^{-\delta \rho}\left[1-(1-2 x)^{2}\right],
$$

where $\rho$ is the number density and $x$ is the proton fraction. In Eq. (2.5), $E_{3}^{\mathrm{R}}(\rho, x) / N$ and $E_{3}^{2 \pi}(\rho, x) / N$ are the expectation values of the repulsive and two- $\pi$-exchange parts of the UIX potential in the case of the Fermi gas, respectively; the last term is a phenomenological correction term. Then, the total energy $E / N$ is the sum of $E_{2} / N$ and $E_{3} / N$, in which the parameters $\alpha, \beta, \gamma$ and $\delta$ are determined so that $E / N$ reproduces the empirical saturation data, i.e., the saturation density $\rho_{0}=0.16 \mathrm{fm}^{-3}$, the saturation energy $E_{0} / N=-16.1 \mathrm{MeV}$, the incompressibility $K=240 \mathrm{MeV}$ and the symmetry energy $E_{\text {sym }} / N=30 \mathrm{MeV}$ [5, 6].

The obtained total energies per nucleon $E / N$ are shown in Fig. 1: Also shown are the results by APR [7]. It is seen that our results are in good agreement with those by APR, especially in the neighborhood of the normal density. At higher densities, the results by APR become lower due to the pion condensation [7]. It is noted that the FHNC calculations are difficult to perform for asymmetric nuclear matter.

\section{VARIATIONAL CALCULATION AT FINITE TEMPERATURE}

In this section, we extend the variational method by Schmidt and Pandharipande (SP) [8] to 
calculate the free energy per nucleon $F / N$ for asymmetric nuclear matter at finite temperatures [5]. In this method, $F / N$ is expressed as

$$
\frac{F}{N}=\frac{E_{\mathrm{T}}}{N}-T \frac{S}{N}
$$

The approximate internal energy $E_{\mathrm{T}} / N$ is the sum of the two-body energy $E_{\mathrm{T} 2} / N$ and the threebody energy $E_{\mathrm{T} 3} / N$. The two-body energy $E_{\mathrm{T} 2} / N$ is the expectation value of $H_{2}$ with the Jastrow wave function at finite temperature $\Psi(T)$, expressed in the two-body cluster approximation. The functional form of $\Psi(T)$ is Eq. (2.2), with $\Phi_{\mathrm{F}}$ replaced by the Fermi-gas wave function at finite temperature, $\Phi_{\mathrm{F}}(T)$, which is specified by the averaged occupation probabilities of protons $n_{\mathrm{p}}(k)$ and of neutrons $n_{\mathrm{n}}(k)$. Here, $n_{i}(k)(i=\mathrm{p}, \mathrm{n})$ is assumed as

$$
n_{i}(k)=\left\{1+\exp \left[\frac{\varepsilon_{i}(k)-\mu_{i}}{k_{\mathrm{B}} T}\right]\right\}^{-1},
$$

with the single particle energy $\varepsilon_{i}(k)$ being parameterized by the effective mass $m_{i}^{*}$. In this study, the so-called frozen-correlation approximation is employed, i.e., the correlation function $f_{i j}$ in $\Psi(T)$ is assumed to be the same as at zero temperature. The three-body energy $E_{\mathrm{T} 3} / N$ is also assumed to be the same as $E_{3} / N$ at zero temperature. The approximate entropy $S / N$ in Eq. (3.1) is also expressed with $n_{i}(k)$ as

$$
\frac{S}{N}=-\frac{k_{\mathrm{B}}}{N} \sum_{i=\mathrm{p}, \mathrm{n} \text { spin }} \sum_{k}\left\{\left[1-n_{i}(k)\right] \ln \left[1-n_{i}(k)\right]+n_{i}(k) \ln n_{i}(k)\right\} .
$$

Then, $F / N$ is minimized with respect to $m_{\mathrm{p}}^{*}$ and $m_{\mathrm{n}}^{*}$.

The obtained free energies of asymmetric nuclear matter at $T=30 \mathrm{MeV}$ are shown in Fig. 2(a). Also shown are the results with the FHNC calculation by Mukherjee [9]. It is seen that the present results are in good agreement with those by Mukherjee. Other thermodynamic quantities such as entropy, pressure and chemical potential, derived from the free energy, are quite reasonable, though not shown in this paper.

Figure 2(b) shows the comparison between $F / N$ in the frozen-correlation approximation and the fully minimized $F / N$. Here, the latter implies that the free energy is minimized with respect to $m_{\mathrm{p}}^{*}, m_{\mathrm{n}}^{*}$ and the correlation function $f_{i j}$ with two constrains, i.e., the extended Mayer's condition and the healing-distance condition. It is seen in this figure that the free energies in the frozencorrelation approximation are in good agreement with those with the full minimization: the validity of the frozen-correlation approximation is confirmed.

\section{CONCLUDING REMARKS}

In this paper, we constructed an EOS for uniform asymmetric nuclear matter at zero and finite temperatures with the variational method, starting from the realistic nuclear Hamiltonian. The obtained free energy and other thermodynamic quantities such as entropy, pressure and chemical potential are important ingredients in the EOS of SN matter. In order to complete a new nuclear EOS table for SN simulations, it is necessary to treat non-uniform nuclear matter and the formation of nuclei at low densities, in addition to uniform nuclear matter. We plan to construct the non-uniform nuclear EOS in the Thomas-Fermi approximation using the present uniform EOS by following the procedure adopted by Shen et al [2]. 

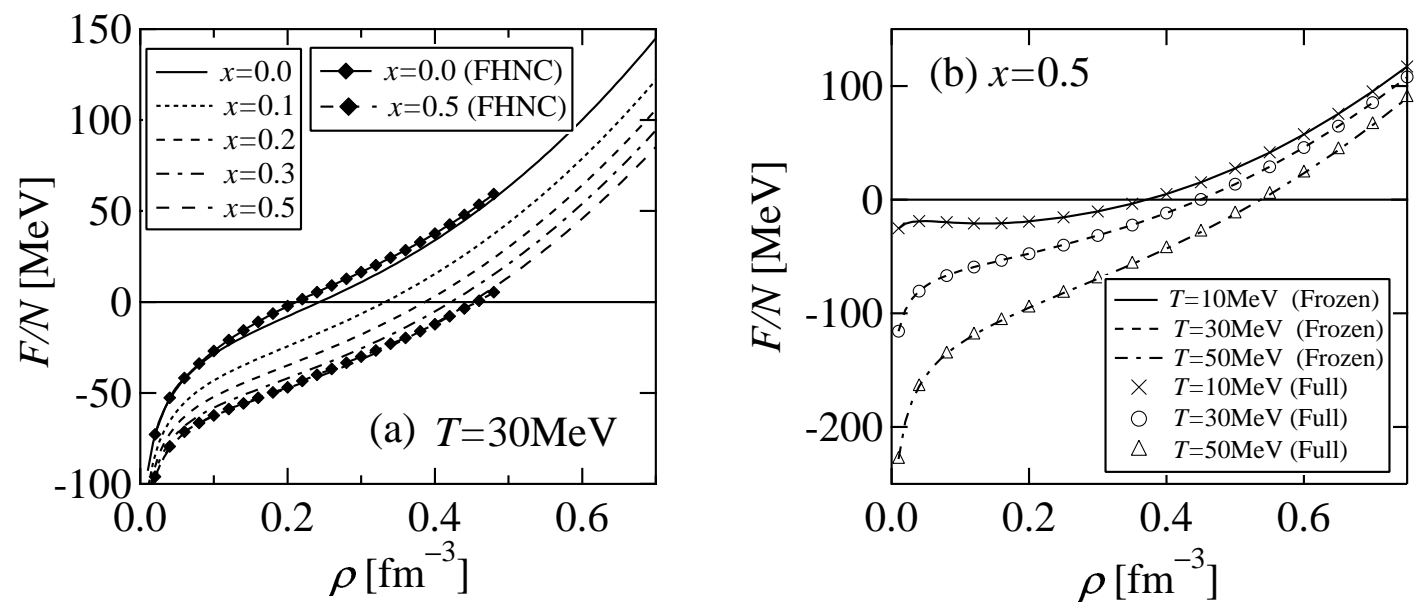

Figure 2: (a) Free energies per nucleon at $T=30 \mathrm{MeV}$ for various proton fractions $x$ as functions of the density $\rho$. Free energies calculated with the FHNC method for symmetric nuclear matter and neutron matter by Mukherjee are also shown. (b) Free energies per nucleon in the frozen-correlation approximation and those with the full minimization as functions of the density $\rho$ for symmetric nuclear matter .

\section{Acknowledgments}

We would like to express special thanks to K. Oyamatsu, K. Sumiyoshi and H. Suzuki for valuable discussions and comments, and A. Mukherjee for providing us with numerical data on free energies with the FHNC calculation. This works is supported by Grants-in-Aid from the Scientific Research Fund of the JSPS (Nos. 18540291 and 21540280) and the Grant-in-Aid for Scientific Research on Innovative Areas (No. 20105004).

\section{References}

[1] J. M. Lattimer and F. D. Swesty, Nucl. Phys. A 535 (1991) 331.

[2] H. Shen, H. Toki, K. Oyamatsu and K. Sumiyoshi, Nucl. Phys. A 637 (1998) 435.

H. Shen, H. Toki, K. Oyamatsu and K. Sumiyoshi, Prog. Theor. Phys. 100 (1998) 1013.

[3] C. Ishizuka, A. Ohnishi, K. Tsubakihara, K. Sumiyoshi and S. Yamada, J. Phys. G 35 (2008) 085201.

[4] K. Nakazato, K. Sumiyoshi and S. Yamada, Phys. Rev. D 77 (2008) 103006.

[5] H. Kanzawa, K. Oyamatsu, K. Sumiyoshi and M. Takano, Nucl. Phys. A 791 (2007) 232.

[6] H. Kanzawa, K. Oyamatsu, K. Sumiyoshi and M. Takano, Prog. Theor. Phys. 122 (2009) 673.

[7] A. Akmal, V. R. Pandharipande and D. G. Ravenhall, Phys. Rev. C 58 (1998) 1804.

[8] K. E. Schmidt and V. R. Pandharipande, Phys. Lett. B 87 (1979) 11.

A. Mukherjee and V. R. Pandharipande, Phys. Rev. C 75 (2007) 035802.

[9] A. Mukherjee, Phys. Rev. C 79 (2009) 045811. 